$\mathbb{T}$ periodica polytechnica

Civil Engineering

$53 / 2$ (2009) $101+106$

doi: 10.3311/pp.ci.2009-2.06

web: http://www.pp.bme.hu/ci

(c) Periodica Polytechnica 2009

RESEARCH ARTICLE

\section{Estimation of CPT resistance based on DPH results}

András Mahler / János Szendefy

Received 2009-02-27, revised 2009-03-24, accepted 2009-04-07

\begin{abstract}
Hungarian experience about the correlation of CPT and DPH results is summarized. A historical review of CPT-DPH and $C P T-S P T$ correlations is presented, and the reliability of the published CPT-DPH correlations is analyzed using recent data from Hungarian geotechnical practice. Based on these data the paper defines soil types where reliable correlation exists and proposes formulas describing the relationships between the CPT and DPH results, because in the case of hard state clays and soils containing gravel an acceptable relationship cannot be stated.
\end{abstract}

\section{Keywords \\ $C P T \cdot D P H \cdot$ tip resistance}

\section{András Mahler}

Department of Geotechnics, BME, Múegyetem rkp. 3. Budapest, H-1521, Hungary

e-mail: mahler@mail.bme.hu

\section{János Szendefy}

Department of Geotechnics, BME, Múegyetem rkp. 3. Budapest, H-1521, Hungary

e-mail: szendefyjano@freemail.hu

\section{Introduction}

The two sorts of the indirect soil exploration methods widely used in the Hungarian geotechnical practice are the dynamic probing (heavy, DPH), and the cone penetration test or piezocone test (CPTu). The dynamic probing has been a popular method for a long time, therefore there is plenty of relationships (local experience) regarding the soil's condition and DPH results. The use of the CPTu has been growing rapidly in the past decades in our country.

It would be useful to find a relationship between the results from both probe types for the science and for engineers involved in the engineering practice being familiar with the evaluation methods for only one of the probe types and knowing less the possibilities of the other type. As the dynamic probing is less expensive, this will presumably come down to have a possibility to determine the approximate value of $q_{c}$ (CPTu cone resistance) from dynamic probing. Although the reliability declines this way, the numerous relationships elaborated for CPTu tests become usable also for DPH results - the dynamic probes can be used more widely thereby.

\section{Overview of the results from earlier investigations}

More investigations were carried out abroad earlier to confront the results from CPT and DPH, it is reasonable therefore to overview and examine the relationships proposed by these before the processing of the Hungarian data. We found during searching in the literature that only few publications [6] can be found about the correlation of the results from the in Hungary most widely used dynamic probe (DPH - Dynamic Probing, Heavy) and the cone penetration test (CPTu). These results come mostly from German speaking countries, because these probe types are widely used there. Far more (and more recent) investigations can be found on the correlation of the results from the in North-America widespread SPT (Standard Penetration Test) and of CPTu. As this problem is very similar to the actual task (the relationship between the results from static and dynamic probes), it is useful to study these works and utilize their experience, too. 
Tab. 1. Correlation between DPH and CPT (Biedermann, 1984)

\begin{tabular}{lccc}
\hline Soil type & $q_{c} / N_{10}$ & $q_{c} / N_{20}$ & Range of validity \\
\hline poorly graded sand & 0.7 & 0.35 & $6<N_{20}<60$ \\
\hline well graded sand & 1.0 & 0.5 & $6<N_{20}<60$ \\
\hline sandy gravel & 1.5 & 0.75 & - \\
\hline clay & 1.0 & 0.5 & $6<N_{20}<38$ \\
\hline
\end{tabular}

\subsection{The correlation between CPTu and DPH}

There has been carried out investigations since the beginning of the dissemination of the cone penetration test, the 1960 decade. This is shown by a publication on this topic of 1968 [5], which proposes for sands the following equation:

$$
q_{c}=4.708 \cdot N_{20} \text {, }
$$

where $q_{c}=\mathrm{CPT}$ cone resistance in $M P a, N_{20}=$ number of blows for $20 \mathrm{~cm}$ penetration of DPH.

This relationship gives a proposal only for sand soils; it tries to describe the correlation between the results from both types of probes by a single factor. We observed that this is valid only for the so called Maihak type CPT, and the author experienced different results from "Dutch" CPT. Although our approximate calculations showed that this equation gives only a very inaccurate approximation of the correlation, a summarizing research report from 1975 [7] contains also this proposal.

A more detailed relationship is proposed by another research report published in 1984 [2], where the author assigns different $q_{c} / N_{10}$ ratios to the different soil types. These values and the corresponding soil types are shown in the following table (1). The table shows the ratios converted to $N_{20}$ and the range of validity for the calculation method, too.

We examined how exactly this relationship describes the correlation between values for both CPT and DPH resistances measured in Hungary. For this reason we derived $q_{c}$ values from DPH results, and represented it as a function of the empirical "measured" resistance on a logarithmic scale (Fig.1).

It can be seen that although the characteristic trend is readable when using this relationship, the data are highly diffuse. This method further-more overestimates $q_{c}$ for clays in every case.

\subsection{The correlation between CPTu and SPT}

The most widespread relationships for the definition of the correlation of the results from these probing methods are those proposed by Robertson et al. (1983) as well as Kulhawy and Mayne (1990). The authors of both methods point up the necessity to correct the results gained from different types of standard penetration tests (SPT). This is based on the fact that different types of devices transmit the dynamic energy to a different extent (efficiency) toward the soil. Earlier measurements of this type were based on the blow numbers pertaining to $60 \%$ efficiency, the results from equipments having less or higher efficiency must be corrected accordingly. This corrected blow num-

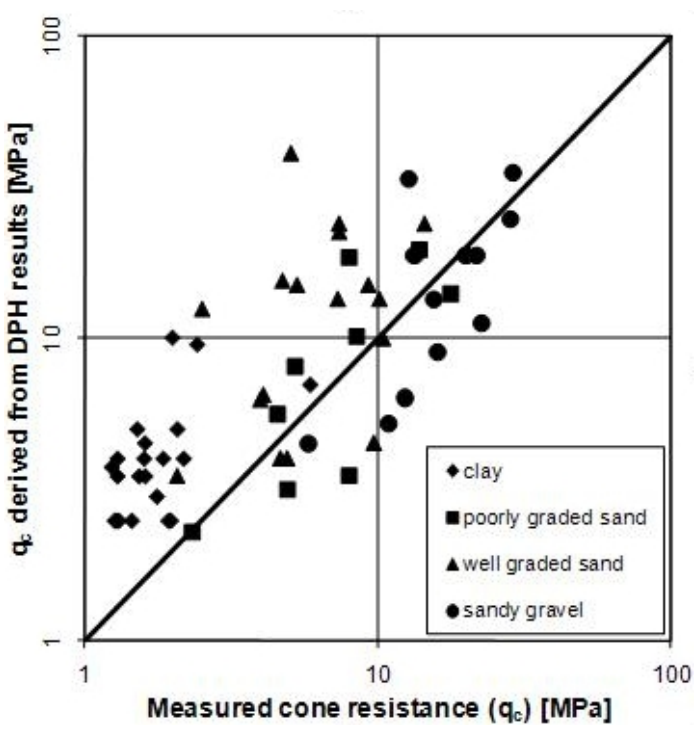

Fig. 1. Measured and derived CPT resistance values

ber is called $N_{60}$. This may be reasonable also in the Hungarian practice of dynamic sounding, but we did not study this question during our investigations because the analyzed DPH tests were carried out using the same device.

Robertson uses in his work earlier already published data gained from 16 locations. He states that the authors of these earlier publications proposed ratios for the results gained from both types of probes spreading a wide range, which seems to be almost inconsistent. This fluctuation of the ratios becomes easily comprehensible nevertheless if their values are plotted as a function of the mean grain size.

The measured $q_{c} / N_{60}$ ratios fit well for a curve if depicted in a semilogarithmic system of coordinates.

Kulhawy and Mayne (1990) propose two relationships in their work. On one hand they improve Robertson's curve by processing further data, on the other hand they propose a new relationship for the $q_{c} / N_{60}$ ratio as a function of the fine content. These two relationships are the followings:

$$
\begin{gathered}
\frac{q_{c} / p_{a}}{N_{60}}=5.44 \cdot d_{50}^{0.26} \\
\frac{q_{c} / p_{a}}{N_{60}}=4.25-\frac{F C}{41.3}
\end{gathered}
$$

where: $p_{a}=$ the reference pressure (equal to atmospherical pressure $=100 \mathrm{kPa}$ ), $N_{60}=$ SPT blow numbers pertaining to $60 \%$ efficiency, $d_{50}=$ the diameter in the grain size distribution curve corresponding to $50 \%[\mathrm{~mm}], F C=$ fine content [\%].

There are lots of publications entertaining this subject besides the ones described above. Their main goal was to analyze the accuracy of the existing methods or to describe local experiences [8].

\section{The correlation between the results of CPTu and DPH}

We utilized the data gained from 83 ground layers of 29 locations to process the Hungarian experiences. On every location boring, CPT and DPH tests were carried out typically until $20 \mathrm{~m}$ 
depth. To filter out the influence of the formation boundaries and interjacent layers we used to our investigations solely data from homogeneous ground layers having the thickness of at least $2 \mathrm{~m}$, and did not take into account the influenced (descending or ascending) probe resistance values measured near the regions of formation boundaries.

According to the preliminary calculations the values of the $q_{c} / N_{20}$ ratios fluctuated within a wide range, but these varying results can be properly separated for non-cohesive and cohesive soils. This is why we studied these both soil types separately as described in the followings.

\subsection{Non-cohesive soils}

As the $q_{c} / N_{20}$ ratios in question are diversified it is useful to depict their values in any case as a function of a third variable (soil parameter). We used for this purpose in case of grained soils and for the SPT-CPT correlations the mean grain size (the inflection point of the grain-size distribution curve) as proposed by Robertson (1983) as well as Kulhawy and Mayne (1990), and the values of the silt+clay content $(d<0.02 \mathrm{~mm}$ according to the Hungarian Standard MSZ 14043-2:1979). We attempted to use also the uniformity coefficient, but this led to a far worse correlation than using the other factors.

A relationship for each case silhouetted well, but the fluctuation of the data was still to high. When examining the different data it became unequivocal that these differences had a trend: the ratios experienced in case of deeper ground layers were situated in the lower part of the set of points while those from ground layers closer to the natural ground level in the upper part of it. This is why we found necessary to correct (divide) the DPH results (blow numbers) by the effective overburden stress, thus the set of points "shrank" close to a curve - i.e. the fluctuation decreased and the relationship became more accurate. In order to get a dimensionless relationship we propose to divide the addends having a pressure type dimension by a reference pressure (according to the atmospherical pressure), thus a dimensionless ("normalized") value, a Normalized CPT-DPH ratio can be generated:

$$
\text { Normalized CPT-DPH ratio }=\frac{q_{c} / p_{a}}{\frac{N_{20}}{\sigma_{v}^{\prime} / p_{a}}}
$$

where: $q_{c}=$ is the CPT cone resistance, $p_{a}=$ the reference pressure (equal to atmospherical pressure $=100 \mathrm{kPa}$ ), $N_{20}=$ number of blows for $20 \mathrm{~cm}$ penetration of $\mathrm{DPH}, \sigma_{v}{ }^{\prime}=$ effective overburden stress

On the following figures we demonstrate the ratios between the CPT cone resistance and the number of blows for $20 \mathrm{~cm}$ penetration of DPH corrected by the effective overburden stress as a function of the silt+clay content (Fig. 2) as well as of the mean grain size (Fig. 3).

Fig. 2 shows that depicting the ratio of the probe resistance as a function of the silt+clay content gives a curve that fits well to the set of points, but the points fluctuate in a relatively

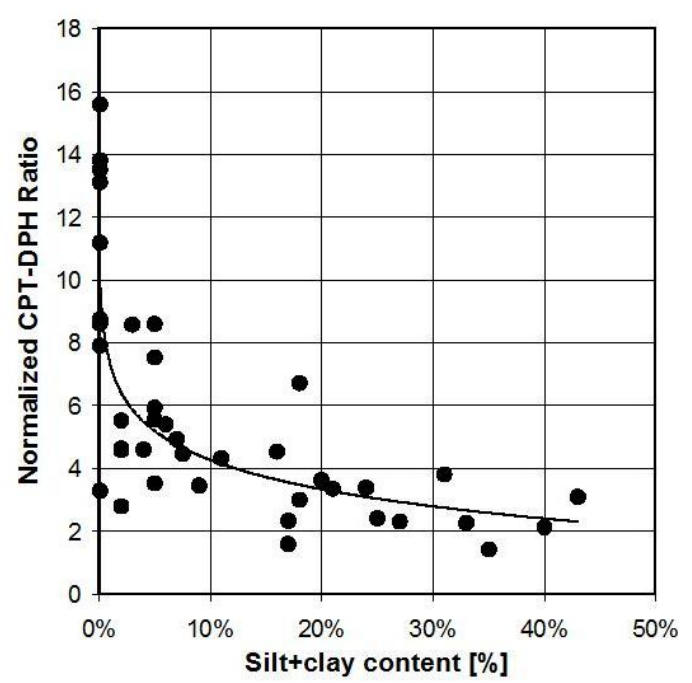

Fig. 2. Normalized CPT-DPH ratio as a function of silt+clay content

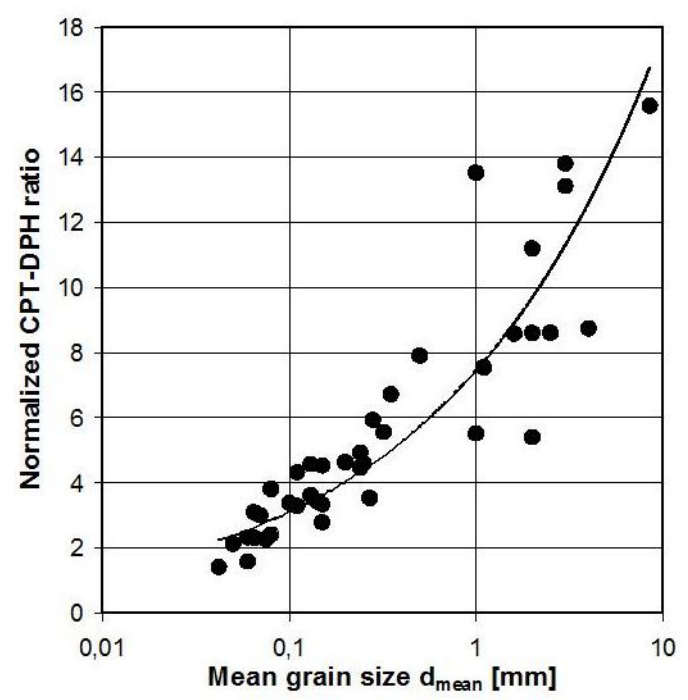

Fig. 3. Normalized CPT-DPH ratio as a function of mean grain size

broad zone, this is also shown by the lower correlation factor of $R^{2}=0.63$. We can observe furthermore that in case of the points according to the silt+clay content $=0 \%$ (these were typically gravelly soils) the ratio varies in a wide range - in this case an explicit relationship cannot be stated.

If we depict this normalized CPT-DPH ratio as a function of the mean grain size, the points according to the $d_{\text {mean }}<1-2 \mathrm{~mm}$ grain size are situated in a very narrow zone, but the bigger mean grain sizes lead to a higher fluctuation of the data here, too. In our opinion the reason for this is that these soils (in the studied cases) contained bigger size gravels, too. The presence of these gravels, the diameter of which exceeds $\sim 1 / 10$ of the diameter of the probe, reduces considerably the reliability (accuracy, repeatability) of the measurements for both probe types this means that the probe resistance varies in a wide range ("jiggles") also in homogenous layers. Of course for these soils also the relationship between the probe types can be determined only with a higher uncertainty, it is useful therefore to handle the soils containing a gravel size fraction $(d>2 \mathrm{~mm})$ separately.

On the following figure (Fig. 4) we marked differently the 


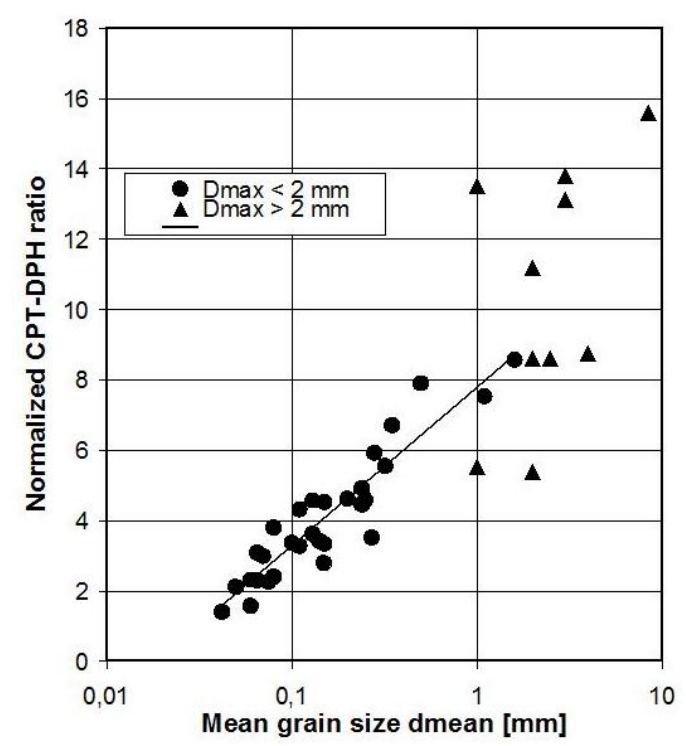

Fig. 4. Normalized CPT-DPH ratio as a function of mean grain size \#2

soils containing a gravel size fraction (triangle) and the ones not containing it (circle). It shows obviously that for gravelly soils it is not possible to determine a reliable relationship between the ratio and the mean grain size (as well as in the case of the silt+clay content). For the case of the soils not containing grains larger than $2 \mathrm{~mm}$ (gravels) the points depicting the ratios fit well to a straight line in a semilogarithmic system of coordinates; this means that the relationship of the probe results can be well described as a logarithmic function of the mean grain size.

We put the best fitting (best correlating) curves for the set of points showed on the figures using the least squares method.

The following formula describes it as a function of silt+clay content:

$$
\frac{q_{c} / p_{a}}{\frac{N_{20}}{\sigma_{v}^{\prime} / p_{a}}}=-2.3 \cdot \log (S+C)+1.88
$$

where $(S+C)=$ the silt + clay content $(d<0.02 \mathrm{~mm})$ of the soil.

Because of the higher fluctuation of the data we propose to use this relationship only for rough estimations.

A more accurate ratio value can be acquired by applying the following relationship using the mean grain size:

$$
\frac{q_{c} / p_{a}}{\frac{N_{20}}{\sigma_{v}^{\prime} / p_{a}}}=4.5 \cdot \log d_{\text {mean }}+7.8
$$

where $d_{\text {mean }}$ is the mean grain size used in the Hungarian practice (,the point of inflection of grain size distribution curve”).

This relationship can be used exclusively for soils not containing grains of diameter $d>2 \mathrm{~mm}$ (gravels). In this case also the correlation coefficient is significantly better than for the former relationship: $R^{2}=0.86$.

\subsection{Cohesive soils}

In the case of cohesive soils the studied problem is more complex and complicated than for non-cohesive soils. During the processing of the results gained from DPH tests we experienced that in homogeneous clay layers following the upper part

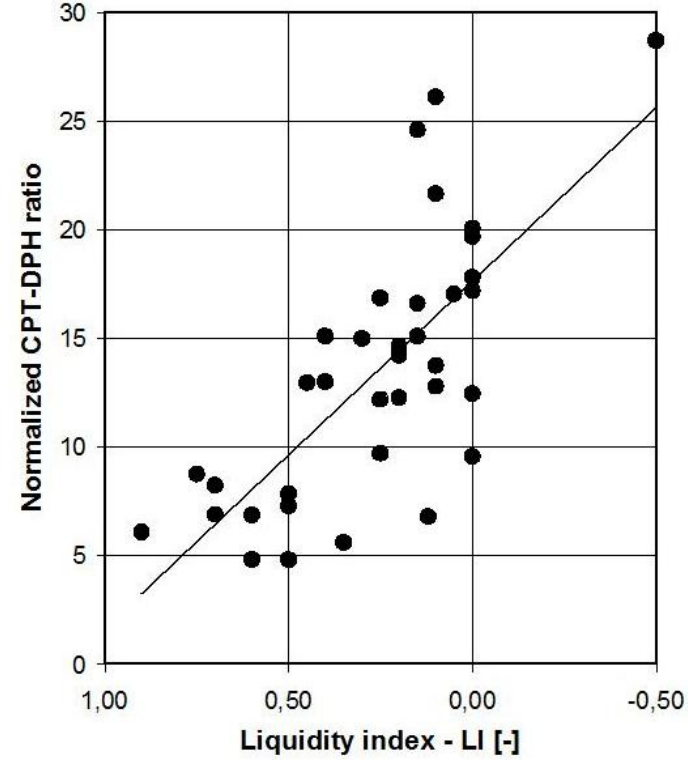

Fig. 5. Normalized CPT-DPH ratio as a function of plasticity index

of $\sim 1.0 \mathrm{~m}$ thickness giving approximately constant probe resistance the blow numbers $\left(N_{20}\right)$ rose often (quasi) linearly as a function of depth, although the type or condition of the soil showed any change neither in the boring nor in the results of the static probe. This phenomenon is caused likely by the fact that because of the dynamic effect the pore-water pressure rises in the clay, the soil does not have enough time to consolidate, the probe "becomes springy" affected by the impacts. Because of all these the blow numbers experienced in clay layers can be hardly evaluated, the attenuation (correction) of the measured values would be necessary in any case. In such cases we considered the upper, nearly constant probe resistance as characteristic for the given layer.

A further problem is affected in the case of cohesive soils by the fact that the excess pore-water pressures caused by both static and dynamic loads are different. While in the case of the static probe (CPTu) the effected pore-water pressure can be measured and therefore taken into account in the calculations, using dynamic probes we haven't any information about the magnitude of the pore-water pressure, which probably changes furthermore during the process of the measurement.

Similarly to the non-cohesive soils we studied the normalized CPT-DPH ratio as a function of a soil parameter also in this case. For cohesive soils it is obvious to use the plasticity index $(P I)$ and the Liquidity index $(L I)$ for this purpose, thus the following figures (Fig. 5 and Fig. 6) demonstrate the normalized CPT-DPH ratio as a function of the plasticity index and the liquidity index.

The figures demonstrate that it is very difficult to find an exact relationship between the values, also the low values of the correlation coefficient underpin this (for $P I R^{2}=0.31$, for $L I$ $\left.R^{2}=0.48\right)$.

Besides these we can see a definite, broader and near linear zone where the points are located. On the figure of the ratios and the plasticity index (Fig. 5) there is a single point which is 


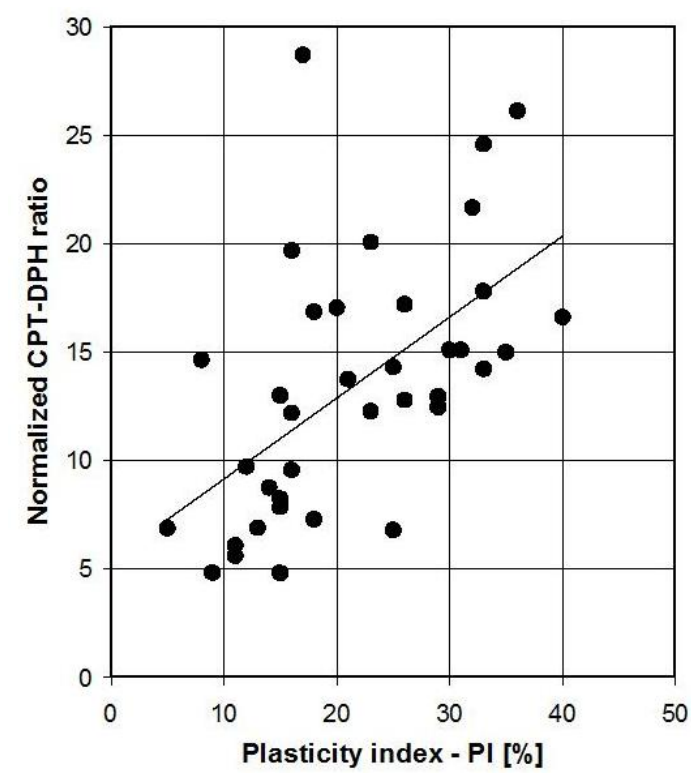

Fig. 6. Normalized CPT-DPH ratio as a function of liquidity index

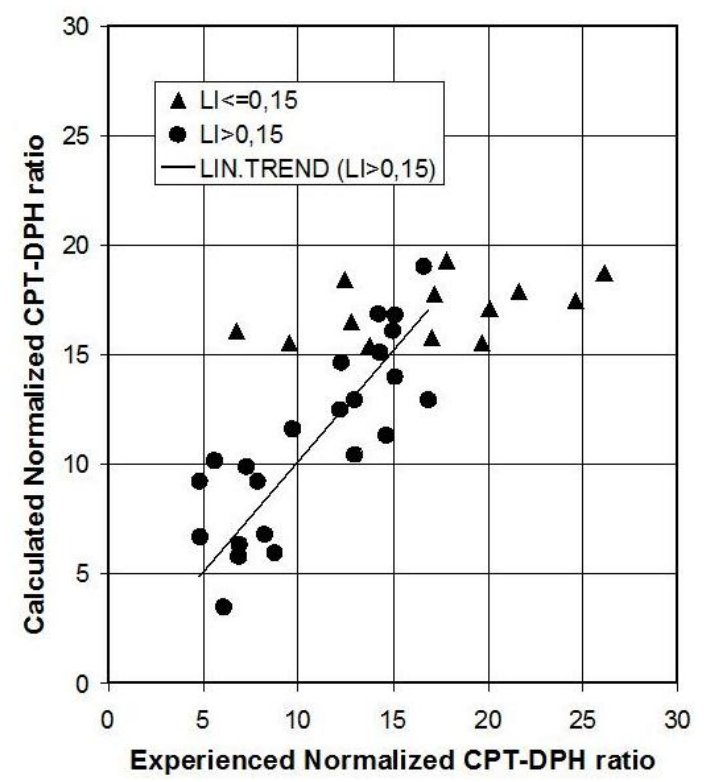

Fig. 7. Experienced vs. calculated normalized CPT-DPH ratio

far out of this zone, this is however not a measuring error, but represents a clay harder than the others $(L I=-0.50)$ while for all other locations $L I \geq 0.00$ ). Also this underpins the statement that the studied normalized CPT-DPH ratio rises when either the plasticity index rise or the liquidity index decrease. To improve the accuracy of the relationship it is necessary to create the formula as a function of these both factors as follows:

$$
\frac{q_{c} / p_{a}}{\frac{N_{20}}{\sigma_{v}^{\prime} / p_{a}}}=0.22 \cdot P I-12.2 \cdot L I+12 .
$$

where $P I=$ plasticity index (in $\%$ ), $L I=$ liquidity index.

To demonstrate the accuracy of the results we show on the next figure (Fig. 7) the values of the normalized CPT-DPH ratio both measured using the test results and calculated by the above formula.

On this figure (Fig. 7) the cohesive soils having lower liquidity index (LI) than 0.15 are represented by triangles, and those with values $L I \geq 0.15$ by circles. It is manifest that the calculated ratio varies between 15 and 20 for stiff cohesive soils, but the measured values spread a more wide range. Therefore for such cohesive soils the proposed relationship is not able to give a reliable result. For softer soils $(L I \geq 0.15)$ the points are situated close to the straight $45^{\circ}$ line representing the exact calculation, thus the proposed relationship gives a good approximation for the ratio of the probe resistance. For such soils the value of the correlation coefficient presented itself as $R^{2}=0.66$, and the experienced standard deviation as $\sigma=2.45$. This can be considered as encouraging taking into account the complex nature of the problem.

\section{Conclusions}

We processed the data gained from both CPT and DPH tests of 83 ground layers on 29 locations. The following conclusions can be drawn:

- In the case of soils containing a gravel size fraction $\left(d_{\max }>\right.$ $2.0 \mathrm{~mm}$ ) an acceptable relationship cannot be stated between the probe resistance values. This is caused by the fact that because of the higher grain size the results fluctuate in a very wide range for both probe types, even in homogenous layers. We could not find any relationship between the high standard deviation CPT and DPH results capable for even rough estimations.

- For harder state clays ( $L I \leq 0.15)$ the conditions of the cohesive soils can not be reliably characterized using DPH. While the CPT resistance was approximately constant in such homogenous clay layers, the DPH blow numbers spread a wide range.

- For the other soil types the relationship between the CPTu and DPH results can be defined in case of grained soils with high reliability, in case of cohesive soils with medium reliability.

\section{References}

1 Akca N, Correlation of SPT-CPT data from the United Arab Emirates, Engineering Geology 67 (2003), no. 3, 219-231, DOI 10.1016/S00137952(02)00181-3.

2 Biedermann B, Vergleichende Untersuchungen mit Sonden in Schluff, Forschungsberichte aus Bodenmechanik und Grundbau (1984).

3 Kulhawy $\mathbf{F H}$, Manual on estimating soil properties for foundation design (1990). Final Report 1493-6.

4 Lunne T, Direct applications of CPT/CPTu results. Cone Penetrometer Testing: Geotechnical and Environmental (2007.02.19.) Lunne Lecture\#5.

5 Melzer KJ, Sondenuntersuchungen in Sand, no. 43. Grundbau und Bodenmechanik an der TH Aachen.

6 Pusztai J, Suggestion to determination of the bearing capacity of piles on the basis of CPT sounding tests 48/1-2 (2004), 39-46.

7 Teferra A, Beziehungen zwischen Reibungswinkel, Lagerungsdichte und Sondierwiderständen nichtbindiger Böden mit verschiedener Kornverteilung, Forschungsberichte aus Bodenmechanik und Grundbau (1975). 
8 Viana da Fonseca A, Carvalho J, Ferreira C, Santos JA, Almeida F, Perreira E, Feliciano J, Grade J, Oliveira A, Characterization of a profile of residual soil from granite combining geological geophysical and mechanical testing techniques, Geotechnical and Geological Engineering, posted on 2006, 1307-1348, DOI 10.1007/s10706-005-2023-z, (to appear in print). 